\title{
Familial thrombophilia and retinal vein occlusion
}

\section{Abstract}

Purpose To perform a pilot study on the prevalence of familial thrombophilia in all cases of retinal vein occlusion with no known risk factors.

Methods Over the 1 year study period 71 patients presented with a new diagnosis of retinal vein occlusion (age 28-90 years). Fiftyfive $(\mathbf{7 7} \%)$ were excluded because of local predisposing factors. The remaining 16 (23\%) had a full risk factor history taken and blood investigations of rheological factors and thrombophilia including tests for the factor $\mathrm{V}$ Leiden mutation, prothrombin G20210A allele and hyperhomocysteinaemia.

Results Of those with no local predisposing factors, 3 patients had antiphospholipid antibodies, 3 had raised fibrinogen levels, 4 had hyperhomocysteinaemia and 1 was heterozygous for the Leiden mutation. Other lifestyle risk factors such as obesity, smoking and a positive family history of venous thrombosis were not uncommon. No patient had the prothrombin G20210A variant. Conclusions It seems likely that several risk factors, both genetic and acquired, need to be present for thrombosis to occur. In investigating a new patient with a retinal vein occlusion one should test for hypertension, glaucoma and diabetes mellitus. Estimation of plasma viscosity and a full blood count are cheap investigations which may reveal neoplasia or vasculitis, and lipid levels should be estimated. In a young patient or one with an unexpected vein occlusion and a personal or family history of thrombosis, a hypercoagulable state may rarely be identified. This additional testing should include testing for antiphospholipid antibodies and a full thrombophilia screen including the factor $\mathrm{V}$ Leiden mutation, homocysteine and the prothrombin variant as part of a clinical trial. Until the role of these markers in thrombosis is better defined in relation to causation of retinal vein occlusion and treatment has been shown to improve outcome, we can not recommend them for routine testing. If a hereditary defect is found, referral should be made to a haematologist and consideration given to anticoagulation and screening of family members to prevent further thrombotic episodes. Retinal vein occlusions are multifactorial in origin except in rare cases.

Key words Factor V Leiden,

Hyperhomocysteinaemia, Management, Prothrombin, Retinal vein occlusion, Thrombophilia, Thrombosis

Venous thrombosis is a multifactorial disease. Risk factor profiles for central retinal vein occlusion (CRVO), hemiretinal vein occlusion (HRVO) and branch retinal vein occlusion (BRVO) have been well documented, with hypertension, diabetes mellitus and primary open angle glaucoma being the most frequently associated. ${ }^{1-5}$ Hyperviscosity and

hypercoagulable states are felt to play a role in retinal vein occlusion, ${ }^{6-8}$ especially in patients under the age of 60 years. ${ }^{9}$ With the increasing recognition of hyperhomocysteinaemia, activated protein $C$ resistance and the prothrombin allele G20210A mutation having a part in thrombosis, we undertook a prospective study to determine the prevalence of these factors in retinal vein occlusion in those patients not having hypertension, open angle glaucoma, diabetes mellitus or carcinoma.

\section{Patients and methods}

Seventy-one patients attending Bradford Royal Infirmary ophthalmic department over a 1 year period were diagnosed as having a retinal vein occlusion (40 men, 31 women; age range 28-90 years: CRVO $(n=42)$, HRVO $(n=2)$, BRVO $(n=27))$. Fifty-five patients were excluded from the study because of local predisposing factors: hypertension $(n=33)$, diabetes mellitus $(n=11)$, primary open angle glaucoma $(n=5)$, raised lipid levels $(n=4)$ and carcinoma $(n=2)$. The remaining 16 patients $(23 \%)$ were all investigated within 6 months after the occurrence of their retinal vein occlusion. All patients in this latter group were normotensive (systolic blood pressure $<160 \mathrm{mmHg}$; diastolic blood $<90 \mathrm{mmHg}$ ) and had no laboratory parameters suggestive of renal or hepatic

\author{
O. Backhouse \\ L. Parapia \\ I. Mahomed \\ D. Lee \\ Departments of \\ Ophthalmology and \\ Haematology \\ Bradford Royal Infirmary \\ and University \\ Bradford, UK \\ O. Backhouse \\ Forge Cottage \\ Kirkby Overblow \\ nr Harrogate \\ North Yorkshire HG3 1HF, \\ UK \\ Tel/fax: $+44(0) 1423$ \\ 879512 \\ e-mail: \\ obackhouse@yahoo.com
}

Presented at the Royal College of Ophthalmologists Annual Congress, Cardiff 1999

Received: 1 February 1999 Accepted in revised form: 25 August 1999 
disease. Ten were non-smokers, 2 ex-smokers (12-15 years) and 4 current smokers (2-40 cigarettes/day). Diagnosis of retinal vein occlusion was made by fundal indirect ophthalmoscopy.

Venous blood parameters measured were: full blood count, plasma viscosity, C-reactive protein, urea and electrolytes, glucose, lipid profile, thyroid function, liver function, autoantibody profile, plasma electrophoresis, homocysteine, fibrinogen, prothrombin time, activated partial thromboplastin time, factor VIII, functional and immune antithrombin III, functional and immune protein C, dilute Russell viper venom test, free protein $S$, activated protein $C$ resistance (APCR) and prothrombin variant (G20210A). If APCR was found, then the factor $V$ Leiden mutation was tested for. All tests were repeated if found to be positive.

In the study group, thrombotic associations documented were: history of smoking, obesity, age, sex, family history of thrombosis, oral contraceptive use, recent surgery, immobilisation, pregnancy/puerperium, and other medical conditions such as vasculitis.

\section{Results}

We found a strong association between retinal vein occlusion and a history of hypertension, diabetes mellitus, open angle glaucoma and carcinoma, which agrees with the findings of other studies.

Sixteen patients were negative for major risk factors (Table 1). Thirteen had non-ischaemic CRVO and 3 had non-ischaemic BRVO. All the venous occlusions were of sudden onset precipitating prompt ophthalmic opinion. Of the 3 patients with BRVO, 2 were under the age of 60 years. The youngest patient had a combination of five risk factors and the other was weakly positive for anticardolipin antibodies. The patient over the age of 60 years had had previous vascular events, was a current smoker and had hyperhomocysteinaemia.
Of the 7 patients under the age of 60 years with CRVO, 3 had no risk factors. Two patients had weak titres of antiphospholipid antibodies, 2 had raised fibrinogen levels, 2 were obsese, 2 had a strong personal or family history of thrombosis, 1 was a smoker, 1 was heterozygous for factor $\mathrm{V}$ Leiden mutation and 1 had hyperhomocysteinaemia. Concurrent medical problems were Raynaud's syndrome in 1 patient.

Of the 6 patients over the age of 60 years with CRVO, 2 had hyperhomocysteinaemia, 2 had no risk factors, 1 was a smoker and 1 had a weak titre of antinuclear antibodies. No patient in the study group had any other thrombophilic markers such as protein $\mathrm{S}$ deficiency.

\section{Discussion}

Data from the Eye Disease Case-Control Study Group identified hypertension and primary open angle glaucoma as major risk factors for retinal vein occlusion. Also associated was diabetes mellitus, but this probably plays a lesser role in BRVO. ${ }^{1-3}$ The greater association of hypertension in BRVO may reflect the effect of hypertension at the crossing of the retinal artery and vein where they share a common adventitial sheath. The characteristic fundal findings occur downstream to this crossing. ${ }^{10}$

Larsson et al. ${ }^{9}$ found that $26 \%$ of patients aged under 50 years with a CRVO had APCR, but no such association was found in those over the age of 50 years. ${ }^{11}$ No confirmatory test for factor $V$ Leiden mutation was performed, though the factor $\mathrm{V}$ Leiden mutation has been reported to account for $94 \%$ of all APCR. ${ }^{12}$ Such a high association of APCR and retinal vein occlusion has not been confirmed by other studies. ${ }^{13-17}$ This mutation is a relatively common hereditary abnormality, with a $1-8 \%$ prevalence of heterozygous carriers, ${ }^{18,19}$ but the prevalence is variable, being high in northern Europe (southern Sweden) and low in Italy, ${ }^{20}$ Asia and Africa. ${ }^{21}$ In this study only 1 of the 6 patients aged under 50 years had the factor V Leiden mutation; she also suffered from

Table 1. Patient details of age, sex, type of vein occlusion and risk factors

\begin{tabular}{|c|c|c|c|c|}
\hline $\begin{array}{c}\text { Patient } \\
\text { no. }\end{array}$ & $\begin{array}{c}\text { Age } \\
\text { (years) }\end{array}$ & Sex & $\begin{array}{l}\text { Vein } \\
\text { occlusion }\end{array}$ & Risk factors \\
\hline 1 & 28 & $\mathrm{~F}$ & BRVO & Homocysteine $20(0-18 \mu \mathrm{mol} / \mathrm{l})$, fibrinogen $4.2(1.5-4 \mathrm{~g} / \mathrm{l})$, smoker, obese, $\mathrm{FH}+\mathrm{ve}$, IHD \\
\hline 2 & 74 & M & CRVO & Homocysteine 24, ex-smoker for 15 years \\
\hline 3 & 74 & $\mathrm{~F}$ & CRVO & Antinuclear antibody weakly + ve, cholesterol 6.9 , hypothyroid \\
\hline 4 & 76 & M & CRVO & Smoker \\
\hline 5 & 49 & M & CRVO & All -ve \\
\hline 6 & 43 & M & BRVO & Anticardiolipin antibody weakly +ve, ex-smoker 15 years \\
\hline 7 & 52 & M & CRVO & Lupus anticoagulant weakly +ve, obese, previous PE \\
\hline 8 & 43 & $\mathrm{~F}$ & CRVO & Fibrinogen 4.4 , smoker, previous PE and DVT, FH +ve, DVT \\
\hline 9 & 79 & M & CRVO & Homocysteine 21 \\
\hline 10 & 68 & M & BRVO & Homocysteine 21 , smoker, previous CVA $\times 2$ and MI \\
\hline 11 & 84 & $\mathrm{~F}$ & CRVO & All -ve \\
\hline 12 & 42 & $\mathrm{~F}$ & CRVO & Fibrinogen, anticardolipin antibody weakly +ve, obese \\
\hline 13 & 62 & $\mathrm{~F}$ & CRVO & All -ve \\
\hline 14 & 36 & $\mathrm{~F}$ & CRVO & Factor V Leiden heterozygous, Raynaud's syndrome \\
\hline 15 & 43 & M & CRVO & All -ve \\
\hline 16 & 51 & M & CRVO & All -ve \\
\hline
\end{tabular}

$\mathrm{BRVO}$, branch retinal vein occlusion; CRVO, central retinal vein occlusion; $\mathrm{FH}$, family history; IHD, ischaemic heart disease; PE, pulmonary embolism; DVT, deep venous thrombosis; CVA, cerebrovascular accident; MI, myocardial infarction. 
Raynaud's syndrome but had no other risk factors. It is important to note that most individuals carrying the defect will not experience a thrombotic event. ${ }^{12}$

Women taking oral contraception have a 4 -fold increase in the risk of venous thrombosis, but this is increased by a multiple of 8 if they are carriers of APCR. The combined effect is that a young woman with APCR who also takes oral contraceptives has a 30-fold increase in the risk of thrombosis. ${ }^{22}$ The Leiden group concluded that routine screening prior to commencing oral contraception is not justifiable, but that it should be carried out if there is a history of thrombosis, to provide information for counselling on the choice of contraception

It is unknown whether patients with venous thrombosis and factor $\mathrm{V}$ Leiden should be anticoagulated and, if so, at what dose and for how long. A calculation of the benefit to risk ratio of oral anticoagulation with a target INR of 2.5 does not support the use of long-term therapy in all patients with the factor $\mathrm{V}$ Leiden mutation following a first venous thrombotic event. ${ }^{23}$ The significance of APCR in the absence of the factor V Leiden mutation remains unclear. ${ }^{24}$

The G20210A prothrombin variant is another common abnormality, with a carrier prevalence of $1-4 \%$; it is more common in southern Europe compared with the north. Like the factor $\mathrm{V}$ Leiden mutation it is very rare in people of Asian or African descent. ${ }^{25}$ The resulting elevated plasma prothrombin levels increases the risk of venous thrombosis. ${ }^{26,27}$ An association of the prothrombin variant and factor $\mathrm{V}$ Leiden in causing arterial disease has not been demonstrated convincingly. ${ }^{28}$ This variant was not found in any patient in the study group.

A variant form of the enzyme methylene tetrahydrofolate reductase is found in its homozygous form in $10 \%$ of the population and may cause raised levels of homocysteine. Hyperhomocysteinaemia predisposes the individual to arterial and venous thrombosis ${ }^{29}$ and has been documented in association with retinal artery and vein occlusions. ${ }^{30,31}$ Increased plasma homocysteine levels may result from enzyme and vitamin deficiencies, smoking, increasing age, medical disorders such as renal failure or as an acute-phase response, and certain medications including thiazide diuretics. ${ }^{32}$ The haematological and vascular abnormalities associated with hyperhomocysteinaemia lead to a proatherogenic and prothrombotic metabolic mileu. ${ }^{33,34}$ Folic acid supplementation, with or without vitamin B12, lowers blood homocysteine levels but largescale randomised trials are now needed to see whether this lowering reduces the risk of vascular disease. ${ }^{35}$ There is preliminary evidence to suggest that this vitamin therapy may prevent progression of cerebral atherosclerosis. $^{36}$ The 4 patients in the study group with hyperhomocysteinaemia were commenced on $5 \mathrm{mg} /$ day folic acid supplementation which reduced the levels of plasma homocysteine. Homocysteine potentiates other risk factors such as hypertension and hyperlipidaemia and raised levels should not be interpreted in isolation. ${ }^{37}$
It is unknown whether the strong link between hyperhomocysteinaemia and cardiovascular events is actually causal. $^{38}$

Antiphospholipid antibodies are a heterogeneous array of antibodies directed against phospholipids. The two most commonly tested for are the anticardolipin antibody and lupus anticoagulant. They have a paradoxical phenomenon of a thrombotic tendency in vivo and prolongation of coagulation in vitro and are associated with arterial and venous thrombosis. ${ }^{39}$ Their incidence increases with age and they may be identified in $14 \%$ of healthy elderly patients. ${ }^{40}$ Thrombotic episodes are recurrent and the site of the first thrombotic event tends to predict the site of subsequent events. ${ }^{41}$ Elevated titres of antiphospholipid antibodies in a healthy person do not warrant systemic anticoagulation, ${ }^{42}$ but in the symptomatic patient optimal treatment is unclear. However, it has recently been found that the combination of idiopathic antiphospholipid antibodies with the factor $\mathrm{V}$ Leiden mutation may further increase hypercoagulability. ${ }^{43}$ As the risk of recurrent thrombosis is high, long-term anticoagulation, in which the INR is maintained at or above 3 , is advisable. ${ }^{44}$ Three patients in the study group had antiphospholipid antibodies. All were aged under 60 years and 2 of them had additional risk factors, the third being an ex-smoker for 15 years. The 2 with additional risk factors were anticoagulated with warfarin.

No abnormalities in the rarer inherited hypercoagulable states such as protein $S$, protein $C$ or antithrombin III deficiency were detected in the study group, which agrees with the findings of previous studies. ${ }^{8,45}$ However, isolated case reports have been documented. ${ }^{46}$

The number of risk factors required to cause thrombosis decreases with age, which itself therefore appears as a strong risk factor for thrombosis. It seems that with aging changes in the vessel wall and possible reduced mobility may all add to the risk profile, necessitating fewer additional risk factors for thrombosis to occur. In children, three or four risk factors are often required to precipitate thrombosis. ${ }^{47}$

It seems likely that several risk factors, both genetic and acquired, need to be present for thrombosis to occur. In investigating a new patient with a retinal vein occlusion one should test for hypertension, glaucoma and diabetes mellitus. Estimation of plasma viscosity and a full blood count are cheap investigations which may reveal neoplasia or vasculitis, and lipid levels should be estimated. In a young patient, or one with an unexpected vein occlusion and a personal or family history of thrombosis, a hypercoagulable state may rarely be identified. This additional testing should include testing for antiphospholipid antibodies and a full thrombophilia screen including the factor $\mathrm{V}$ Leiden mutation, homocysteine and the prothrombin variant, as part of a clinical trial. Until the role of these markers in thrombosis is better defined in relation to causation of retinal vein occlusion and treatment shown to improve outcome, we can not recommend them for routine testing. If an 
hereditary defect is found, referral should be made to a haematologist and consideration given to anticoagulation and screening of family members to prevent further thrombotic episodes. Retinal vein occlusions are multifactorial in origin except in rare cases.

\section{References}

1. The Eye Disease Case-Control Study Group. Risk factors for branch retinal vein occlusion. Am J Ophthalmol 1993;116:286-96.

2. The Eye Disease Case-Control Study Group. Risk factors for central retinal vein occlusion. Arch Ophthalmol 1996;114:545-54.

3. The Eye Disease Case-Control Study Group. Risk factors for hemiretinal vein occlusion: comparison with risk factors for central and branch retinal vein occlusion. Ophthalmology 1998;105:765-71.

4. Rath EZ, Frank RN, Shin DH, et al. Risk factors for retinal vein occlusions: a case-control study. Ophthalmology 1992;99:509-14.

5. Appiah AP, Trempe CL. Risk factors associated with branch vs central retinal vein occlusion. Ann Ophthalmol 1989;21:153-7.

6. Glacet-Bernard A, Chabanel A, Lelong F, et al. Elevated erythrocyte aggregation in patients with CRVO with and without conventional risk factors. Ophthalmology 1994;101:1483-7.

7. Arend O, Remky A, Jung F, et al. Role of rheologic factors in patients with acute central retinal vein occlusion. Ophthalmology 1996;103:80-6.

8. Bandello F, Vigano D'Angelo S, Parlavecchia M, et al. Hypercoagulability and high lipoprotein (a) levels in patients with central retinal vein occlusion. Thromb Haemost 1994;72:39-43.

9. Larsson J, Olafsdottir E, Bauer B. Activated protein C resistance in young adults with central retinal vein occlusion. Br J Ophthalmol 1996;80:200-2.

10. Kumar B, Yu D, Morgan W, et al. The distribution of angioarchitectural changes within the vicinity of the arteriovenous crossing in branch retinal vein occlusion. Ophthalmology 1998;105:424-7.

11. Larsson J, Sellman A, Bauer B. Activated protein C resistance in patients with central retinal vein occlusion. $\mathrm{Br} \mathrm{J}$ Ophthalmol 1997;81:832-4.

12. Zoller B, Svensson PJ, He X, et al. Identification of the same factor $\mathrm{V}$ gene mutation in 47 out of 50 thrombosis-prone families with inherited resistance to activated protein C. J Clin Invest 1994;94:2521-4.

13. Graham SL, Goldberg I, Murray B, et al. Activated protein C resistance: low incidence in glaucomatous optic disc haemorrhage and central retinal vein occlusion. Aust NZ J Ophthalmol 1996;24:199-205.

14. Hodgkins PR, Perry DJ, Sawcer SJ, et al. Factor V and antithrombin gene mutations in patients with idiopathic central retinal vein occlusion. Eye 1995;9:760-2.

15. Raguenes O, Mercier B, Escoffre M, et al. $1691 \mathrm{G}$ to A mutation of the factor $\mathrm{V}$ gene: no association with thrombosis of the central retinal vein. Presse Med 1996;25:460.

16. Linna T, Ylikorkala A, Kontula $K$, et al. Prevalence of factor V Leiden in young adults with retinal vein occlusion. Thromb Haemost 1997;77:214-5.

17. Gottlieb JL, Blice JP, Mestichelli B. Activated protein C resistance, factor $\mathrm{V}$ Leiden and central retinal vein occlusion in young adults. Arch Ophthalmol 1998;116:577-9.

18. Bertina RM, Koeleman BP, Koster T, et al. Mutation in blood coagulation factor $\mathrm{V}$ associated with resistance to activated protein C. Nature 1994;369:64-7.
19. Bowen DJ, Bowley S, John M, et al. Factor V Leiden (G1691A), the prothrombin 3'-untranslated region variant (G20210A) and thermolabile methylenetetrahydrofolate reductase (C677T): a single genetic test genotypes all three loci. Determination of frequencies in the $\mathrm{S}$. Wales population of the UK. Thromb Haemost 1998;79:949-54.

20. Cox MJ, Rees DC, Martinson JJ, et al. Evidence for a single origin of factor V Leiden. Br J Haematol 1996;92:1022-5.

21. Rees DC, Cox M, Clegg JB. World distribution of factor V Leiden. Lancet 1995;346:1133-4.

22. Vandenbroucke JP, Koster T, Briet E, et al. Increased risk of venous thrombosis in oral contraceptive users who are carriers of factor V Leiden mutation. Lancet 1994;344:1453-7.

23. Baglin $C$, Brown $K$, Luddington $R$, et al. Risk of recurrent venous thromboembolism in patients with the factor $\mathrm{V}$ Leiden (FVR506Q) mutation: effect of warfarin and prediction by precipitating factors. Br J Haematol 1998;100:764-8.

24. Clark P, Brennand J, Conkie JA, et al. Activated protein C sensitivity, protein $C$, protein $S$ and coagulation in normal pregnancy. Thromb Haemost 1998;79:1166-70.

25. Rosendaal FR, Doggen CJ, Zivelin A, et al. Geographic distribution of the $20210 \mathrm{G}$ to A prothrombin variant. Thromb Haemost 1998;79:706-8.

26. Poort SR, Rosendaal FR, Reitsma PH, et al. A common genetic variation in the 3'-untranslated region of the prothrombin gene is associated with elevated plasma prothrombin levels and an increase in venous thrombosis. Blood 1996;88:3698-703.

27. Hillarp A, Zoller B, Svensson PJ, et al. The 20210A allele of the prothrombin gene is a common risk factor among Swedish outpatients with verified deep venous thrombosis. Thromb Haemost 1997;78:990-2.

28. Longstreth WT, Rosendaal FR, Siscovick DS, et al. Risk of stroke in young women and two prothrombotic mutations: factor V Leiden and prothrombin gene variant (G20210A). Stroke 1998;29:577-80.

29. McCully KS. Homocysteine and vascular disease. Nature Med 1996;2:386-9.

30. Wenzler EM, Rademakers AJ, Boers GH, et al. Hyperhomocysteinaemia in retinal artery and retinal vein occlusion. Am J Ophthalmol 1993;115:162-7.

31. Biousse V, Newman NJ, Sternberg P. Retinal vein occlusion and transient monocular visual loss associated with hyperhomocystinemia. Am J Ophthalmol 1997;124:257-60.

32. Stein JH, McBride PE. Hyperhomocysteinemia and atherosclerotic vascular disease. Arch Intern Med 1998;158:1301-6.

33. Mayer EL, Jacobsen DW, Robinson K. Homocysteine and coronary atherosclerosis. J Am Coll Cardiol 1996;27:517-27.

34. Rees MM, Rodgers GM. Homocysteinemia: association of a metabolic disorder with vascular disease and thrombosis. Thromb Res 1993;71:337-59.

35. Homocysteine Lowering Trialists' Collaboration. Lowering blood homocysteine with folic acid based supplements: meta-analysis of randomised trials. BMJ 1998;316:894-8.

36. Peterson JC, Spense JD. Vitamins and progression of atherosclerosis in hyperhomocysteinaemia. Lancet 1998;351:263.

37. Graham IM, Daly LE, Refsum HM, et al. Plasma homocysteine as a risk factor for vascular disease. JAMA 1997;277:1775-81.

38. Still RA, McDowell IF. Clinical implications of plasma homocysteine measurements in cardiovascular disease. J Clin Pathol 1998;51:183-8.

39. Asherson RA, Khamashta MA, Ordi-Ros J, et al. The 'primary' antiphospholipid syndrome: major clinical and serological features. Medicine 1989;68:366-74. 
40. Vila P, Hernandez MC, Lopez-Fernandez MF, et al. Prevalence, follow-up and clinical significance of the anticardolipin antibodies in normal subjects. Thromb Haemost 1994;72:209-13.

41. Rosove $\mathrm{MH}$, Brewer PM. Antiphospholipid thrombosis: clinical course after the first thrombotic event in 70 patients. Ann Intern Med 1992;117:303-8.

42. Boumpas DT, Fessler BJ, Austin HA, et al. Systemic lupus erythematosus: emerging concepts. 2. Dermatologic and joint disease, the antiphospholipid antibody syndrome, pregnancy and hormonal therapy, morbidity and mortality and pathogenesis. Ann Intern Med 1995;123:42-53.
43. Ames PR, Tommasino C, E'Andrea G, et al. Thrombophilic genotypes in subjects with idiopathic antiphospholipid antibodies: prevalence and significance. Thromb Haemost 1998;79:46-9.

44. Khamashta MA, Cuadrado MJ, Mujic F, et al. The management of thrombosis in the antiphospholipid syndrome. N Engl J Med 1995;332:993-7.

45. Guareschi G, Vigano D'Angelo S, Foresti V. Normal activities of protein $\mathrm{C}$, protein $\mathrm{S}$ and antithrombin III in young patients with retinal vein occlusion. Haematologica 1990;75:483-4.

46. Chung MM, Trese MT, Hong YJ. Protein C levels in retinal vein occlusions. Invest Ophthalmol Vis Sci 1989;30:477.

47. Rosendaal FR. Thrombosis in the young: epidemiology and risk factors. A focus on venous thrombosis. Thromb Haemost 1997;78:1-6. 